Article

\title{
Analysis on Displacement Angle of Phase-Shifted Carrier PWM for Modular Multilevel Converter ${ }^{\dagger}$
}

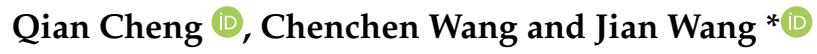 \\ School of Electrical Engineering, Beijing Jiaotong University, Beijing 100044, China; 17117415@bjtu.edu.cn (Q.C.); \\ chchwang@bjtu.edu.cn (C.W.) \\ * Correspondence: jwang4@bjtu.edu.cn \\ + This paper is an extended version of our paper published in 2019 IEEE Energy Conversion Congress and \\ Exposition (ECCE), Baltimore, MD, USA, 2019; pp. 4801-4807.
}

Received: 18 November 2020; Accepted: 17 December 2020; Published: 21 December 2020

check for updates

\begin{abstract}
This paper provides theoretical and experimental discussions on the characteristics of the modular multilevel converter (MMC) when phase-shifted carrier sinusoidal pulse-width modulation (PSC-SPWM) is applied. Harmonic-cancellation characteristics of output voltage and circulating current are analyzed on the basis of a general implementation of PSC-SPWM with two freedom displacement angles. Five available PSC-SPWM schemes with different carrier displacement angles were obtained, and a detailed performance comparison about output voltage and circulating current harmonic characteristics is presented. On the basis of the equivalent circuit with ideal transformer representation of the SMs, capacitor voltages affected by PSC-SPWM schemes are also briefly analyzed. The proposed PSC-SPWM schemes can unify two different cases of odd and even SM situations for output voltage and circulating current harmonic minimization, respectively. Lastly, the optimal schemes for practical MMC application were verified by simulation and experiments on an MMC prototype.
\end{abstract}

Keywords: modular multilevel converter (MMC); phase-shifted carrier SPWM (PSC-SPWM); displacement angle; harmonic characteristics; capacitor voltage

\section{Introduction}

The modular multilevel converter (MMC) has been widely studied because it presents great advantages such as a transformer-less and modular structure, common DC bus, and good harmonic characteristics [1-3]. These advantages make MMC the most attractive topology for various mediumand high-voltage, high-power applications, such as high-voltage direct-current (HVDC) transmission systems [4,5], static synchronous compensators (STATCOM) [6,7], high-voltage isolated DC/DC [8], and medium-voltage motor drive [9-12].

The MMC allows for the flexible selection of inserted submodules (SMs), so there are various modulation methods that directly affect the harmonic characteristics of output voltage and circulating current. The staircase wave-modulation methods in [13] are preferable in applications that require an extremely high number of SMs, such as HVDC applications. Some optimization schemes for reducing power losses are mentioned in $[14,15]$. The selective harmonic eliminating modulation technology [16], space vector pulse-width modulation (SVPWM) [17,18], and phase-shifted carrier sinusoidal pulse-width modulation (PSC-SPWM) technology [19-23] are also considered as modulation methods for MMC applications that need fewer submodules. Among these methods, PSC-SPWM technology is more used for fewer SMs. Each power unit has the same switching frequency with PSC-SPWM, which is a benefit for heat-dissipation structure design. When PSC-SPWM is applied, the output voltage of MMC, as shown in Figure 1, has $N+1$ or $2 N+1$ levels with different 
displacement-angle assignments between the carriers of each SM [21]. The displacement angle affects the harmonic characteristics of output voltage and circulating current. An available implementation scheme of PSC-SPWM technology for MMC was presented in [22]. The displacement angle is set to $\pi / N$ when $N$ is even, and 0 when $N$ is odd for maximal harmonic cancellation of output voltage. On the other hand, to achieve the greatest harmonic cancellation of circulating current, the displacement angle should be $\pi / N$ when $N$ is odd, and 0 when $N$ is even. However, the angle irregularly changes according to the parity of SM number. Furthermore, how the displacement angle between the adjacent SMs in the same arm influences the performance of harmonics and the voltage-balancing issue of capacitors is not considered. Energy distribution is only relatively balanced between submodules when the right displacement angle is selected. Four available PSC-SPWM schemes were presented with performance comparison about output voltage and circulating current harmonic characteristics in our previous paper [23], whereas the selection of displacement angle does not give theoretical derivation.

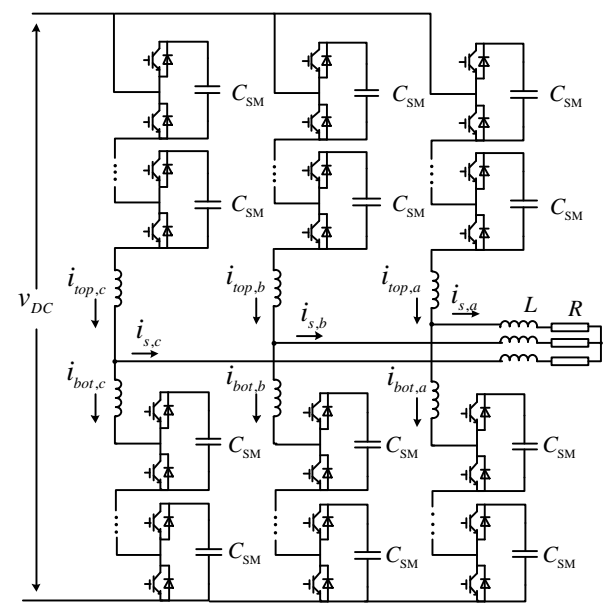

Figure 1. Structure of three-phase modular multilevel converter (MMC).

This paper presents a theoretical derivation of displacement-angle selection based on harmonic-cancellation characteristics. All possible solutions for harmonic cancellation were obtained, and the optimal PSC-SPWM schemes were selected on the basis of harmonic-cancellation characteristics and voltage stability. The proposed PSC-SPWM schemes unify two different cases of odd and even SM situations. The rest of the paper is organized as follows: In Section 2, the MMC topology is introduced, and the mathematical model is established. Then, a general PSC-SPWM scheme with two freedom displacement angles (displacement angle between adjacent SMs in the same arm, and the displacement angle between top and bottom SMs) is presented in Section 3, and optimal PSC-SPWM schemes for MMC were obtained on the basis of mathematical analysis of the harmonic-cancellation characteristics of output voltage and circulating current affected by the displacement angles. Moreover, the capacitor voltage of SMs affected by displacement angles was analyzed on the basis of the equivalent circuit with the ideal transformer representation of the SMs in this section. Section 4 gives the simulation and experiment results with a detailed performance comparison about the characteristics of output voltage and circulating current, which show that the selected displacement-angle assignment was the most suitable PSC-SPWM scheme for practical MMC application. Lastly, conclusions are summarized in Section 5 .

\section{Basic MMC Operational Principle}

\subsection{Structure}

A schematic diagram of a three-phase MMC is shown in Figure 1. The main circuit consists of six arms. Each arm is composed of $N$ half-bridge SMs, which are connected with a separated arm 
inductor in series. Each SM is formed by a DC capacitor $C_{\mathrm{SM}}$ and two power switches. Three-phase RL load is respectively connected to the midpoint of three phases.

\subsection{Model of $M M C$}

The mathematical model of MMC can be obtained by Kirchhoff's voltage law and Kirchhoff's current law as

$$
\left\{\begin{array}{l}
v_{D C}=v_{t o p, j}^{s u b}+v_{b o t, j}^{s u b}+2 L_{a r m} \frac{d i_{z, j}}{d t} \\
v_{s, j}=\frac{1}{2}\left(v_{b o t, j}^{s u b}-v_{t o p, j}^{s u b}\right)-\frac{1}{2} L_{a r m} \frac{d i_{s, j}}{d t},
\end{array}\right.
$$

where $v_{t o p, j}^{s u b}, v_{b o t, j}^{s u b}$ are voltages across $N$ top SMs and bottom SMs in phase $j(j \in\{a, b, c\})$, respectively. $v_{s, j}, i_{s, j}$ are the output voltage and current of phase $j$, respectively. $L_{\text {arm }}$ is arm inductance, and $v_{D C}$ is the DC-link voltage. The circulating current circulates through both the top and bottom arms of phase $j$, which is expressed as

$$
i_{z, j}=\frac{1}{2}\left(i_{t o p, j}+i_{b o t, j}\right)
$$

where $i_{t o p, j}, i_{b o t, j}$ are the top and bottom arm current of phase $j$, respectively.

The voltage across arm inductors $v_{L, j}$ is :

$$
v_{L, j}=2 L_{a r m} \frac{d i_{z, j}}{d t}=v_{D C}-\left(v_{t o p, j}^{s u b}+v_{b o t, j}^{s u b}\right)
$$

For the SMs of MMC, the output voltage of the $k$ th SM in the top and bottom arms can be expressed by $u_{t o p, j}(k), u_{b o t, j}(k)$ respectively:

$$
\left\{\begin{array}{l}
u_{t o p, j}(k)=S_{t o p, j}(k) v_{t o p, j}(k) \\
u_{b o t, j}(k)=S_{b o t, j}(k) v_{t o p, j}(k),
\end{array}\right.
$$

where $v_{t o p, j}(k), S_{t o p, j}(k)$ and $v_{b o t, j}(k), S_{b o t, j}(k)$ are the capacitor voltage and switching function of $k$ th $\mathrm{SM}$ of the top and bottom arms, respectively.

The dynamic equation of SM capacitors can be obtained as

$$
\left\{\begin{array}{l}
C \frac{d v_{t o p, j}(k)}{d t}=S_{t o p, j}(k) i_{z, j}+0.5 S_{t o p, j}(k) i_{s, j} \\
C \frac{d v_{b o t, j}(k)}{d t}=S_{b o t, j}(k) i_{z, j}-0.5 S_{b o t, j}(k) i_{s, j}
\end{array}\right.
$$

According to (1), (4), and (5), one phase circuit of MMC can be redrawn by the equivalent circuit on the basis of the ideal transformer representation of SMs [24], as shown in Figure 2. The notation of turns-ratio term is abbreviated as the switching function of SMs, which fully represents the switching action. On the basis of the equivalent circuit, energy was transferred from the DC side to the AC side through SM capacitors, which acted as intermediate storage elements. In this equivalent circuit, external and internal characteristics of the MMC could be reflected well. 


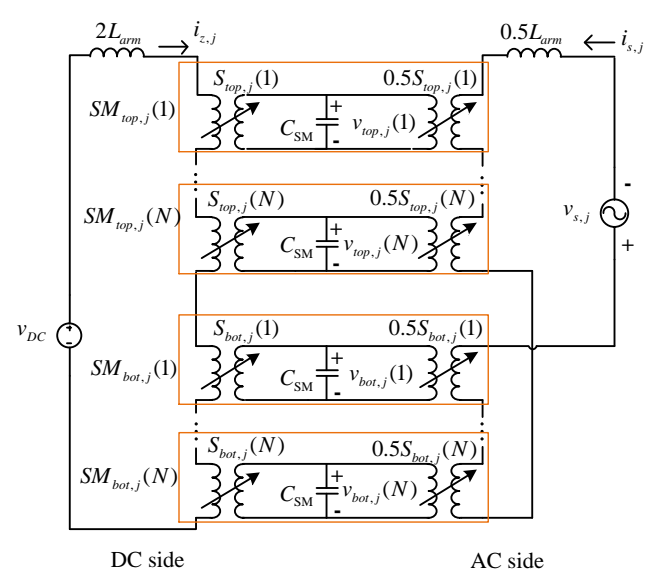

Figure 2. Equivalent circuit of single MMC phase.

\section{Phase-Shifted PWM Schemes for MMC}

In general, the PSC-SPWM scheme of MMC ( $N$ SMs each arm) with two freedom displacement angles is shown in Figure 3. Each SM is assigned with a specific reference signal and a triangular carrier with a displacement angle. The state of the SM is determined by the relationship between reference signal and carrier. When the reference is larger than the carrier value, the corresponding switching function $S_{t o p, j}(k)$ or $S_{b o t, j}(k)$ equals to 1 ; when the reference is smaller than the carrier value, the corresponding switching function $S_{t o p, j}(k)$ or $S_{b o t, j}(k)$ equals to 0 .

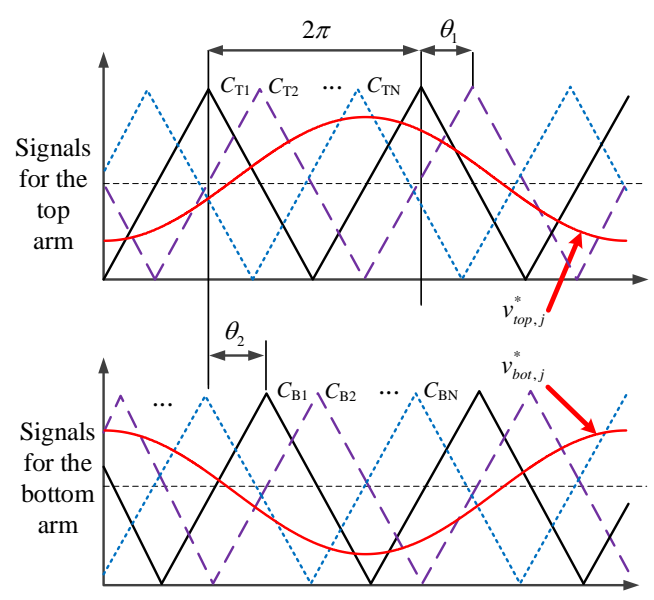

Figure 3. General MMC phase-shifted carrier sinusoidal pulse-width modulation (PSC-SPWM) schemes.

$\theta_{1}, \theta_{2}$ are the displacement angles between the adjacent carriers of the same arm, and the displacement angle between the carriers of the top and bottom arms, respectively. Reference voltages are given as

$$
\left\{\begin{array}{l}
v_{t o p, j}^{*}=\frac{v_{D C}}{2 N}-\frac{M v_{D C}}{2 N} \cos \left(\omega_{0} t+\varphi_{j}\right) \\
v_{b o t, j}^{*}=\frac{v_{D C}}{2 N}+\frac{M v_{D C}}{2 N} \cos \left(\omega_{0} t+\varphi_{j}\right)
\end{array}\right.
$$

where $M$ is the modulation index, $\omega_{0}$ is the fundamental angle frequency, $\varphi_{j}$ is the phase angle, and $v_{D C}$ is the voltage of DC bus.

Each SM corresponding to a specific triangular carrier with different displacement angle. The general carrier function of the top and bottom SMs can be calculated as:

$$
f_{\mathrm{CT}}(k)=\frac{v_{D C}}{N}\left(\frac{1}{2}+\frac{1}{2} \cdot \frac{2}{\pi} \arcsin \left[\sin \left(\omega_{c} t+(k-1) \theta_{1}\right)\right]\right)
$$




$$
f_{\mathrm{CB}}(k)=\frac{v_{D C}}{N}\left(\frac{1}{2}+\frac{1}{2} \cdot \frac{2}{\pi} \arcsin \left[\sin \left(\omega_{c} t+(k-1) \theta_{1}+\theta_{2}\right)\right]\right)
$$

\subsection{Fourier Series Representation of Switching Functions}

The double Fourier series based on analysis in [25] is presented to obtain the harmonic feature of output voltage and circulating current of MMC. In (7) and (8), there are two freedom displacement degrees, $\theta_{1}$ and $\theta_{2}$, which constitute different PSC-SPWM schemes. The principle of how $\theta_{1}$ and $\theta_{2}$ affect the harmonics of output voltage and circulating current is presented in this section.

From (6-8), the switching function can be expressed by Fourier representation as

$$
\begin{aligned}
S_{t o p, j}(k) & =\frac{1}{2}-\frac{M}{2} \cos \left(\omega_{0} t+\varphi_{j}\right)+\sum_{m=1}^{\infty} \sum_{n=-\infty}^{\infty} \frac{2}{m \pi} \\
& \times \sin \left[\frac{(m+n) \pi}{2}\right] \times J_{n}\left(\frac{M m \pi}{2}\right) \\
& \times \cos \left[m\left(\omega_{c} t+(k-1) \theta_{1}\right)+n\left(\omega_{0} t+\varphi_{j}\right)\right] \\
S_{b o t, j}(k)= & \frac{1}{2}+\frac{M}{2} \cos \left(\omega_{0} t+\varphi_{j}\right)+\sum_{m=1}^{\infty} \sum_{n=-\infty}^{\infty} \frac{2}{m \pi} \\
\times & \sin \left[\frac{(m+n) \pi}{2}\right] \times J_{n}\left(\frac{M m \pi}{2}\right) \\
\times & \cos \left[m\left(\omega_{c} t+(k-1) \theta_{1}+\theta_{2}\right)+n\left(\omega_{o} t+\varphi_{j}+\pi\right)\right]
\end{aligned}
$$

where $m$ is carrier index variable $(m=1,2, \ldots, \infty), n$ is the baseband index variable $(n=-\infty, \ldots,-1$, $0,1, \ldots, \infty)$, and $J_{n}(x)$ is the Bessel coefficient.

For simplicity, it was assumed that all the capacitor voltage of SMs are naturally balanced and the voltage fluctuation is ignored. From (1), (9), and (10), the equivalent output voltage of phase $j$ can be obtained as

$$
\begin{aligned}
u_{s, j} & =\frac{M v_{D C}}{2} \cos \left(\omega_{o} t+\varphi_{j}\right)-\frac{1}{2} \sum_{m=1}^{\infty} \sum_{n=-\infty}^{\infty} \frac{2 v_{D C}}{m \pi N} \\
& \times \sin \left[\frac{(m+n) \pi}{2}\right] \times J_{n}\left(\frac{M m \pi}{2}\right) \\
& \times 2 \sin \left[\frac{m \theta_{2}+n \pi}{2}\right] \times \sum_{k=1}^{N} \cos \left(\frac{m(N-1-2(k-1)) \theta_{1}}{2}\right) \\
& \times \sin \left[m \omega_{c} t+n\left(\omega_{0} t+\varphi_{j}\right)+\frac{m \theta_{2}+n \pi}{2}+\frac{m(N-1) \pi}{2}\right]
\end{aligned}
$$

From (11), the harmonic characteristic of output voltage is affected by the following items, which are the function of $\theta_{1}, \theta_{2}$ and $N$ :

$$
\left\{\begin{array}{l}
k_{1}=\sum_{k=1}^{N} \cos \left(\frac{m(N-1-2(k-1)) \theta_{1}}{2}\right) \\
k_{2}=\sin \left[\frac{(m+n) \pi}{2}\right] \\
k_{3}=\sin \left[\frac{m \theta_{2}+n \pi}{2}\right]
\end{array}\right.
$$


Voltage across the arm inductors of phase $j$ can be obtained as

$$
\begin{aligned}
u_{L, j}(k) & =\sum_{m=1}^{\infty} \sum_{n=-\infty}^{\infty} \frac{2 v_{D C}}{m \pi N} \times J_{n}\left(\frac{M m \pi}{2}\right) \times \sin \left[\frac{(m+n) \pi}{2}\right] \\
& \times 2 \cos \left[\frac{m \theta_{2}+n \pi}{2}\right] \times \sum_{k=1}^{N} \cos \left(\frac{m(N-1-2(k-1)) \theta_{1}}{2}\right) \\
& \times \cos \left[m \omega_{c} t+n\left(\omega_{o} t+\varphi_{j}\right)+\frac{m \theta_{2}+n \pi}{2}+\frac{m(N-1) \pi}{2}\right]
\end{aligned}
$$

Similarly, the harmonic characteristic of voltage across the arm inductors is affected by

$$
\left\{\begin{array}{l}
k_{1}=\sum_{k=1}^{N} \cos \left(\frac{m(N-1-2(k-1)) \theta_{1}}{2}\right) \\
k_{2}=\sin \left[\frac{(m+n) \pi}{2}\right] \\
k_{4}=\cos \left[\frac{m \theta_{2}+n \pi}{2}\right]
\end{array}\right.
$$

\subsection{Harmonic-Cancellation Characteristics of MMC with PSC-SPWM}

According to (12) and (14), if one of $k_{1}, k_{2}$ and $k_{3}$ is equal to 0 , the corresponding output-voltage harmonic components $\left(m \omega_{c}+n \omega_{0}\right)$ can be completely eliminated. The corresponding circulating current harmonic components can be completely eliminated if one of $k_{1}, k_{2}$ and $k_{4}$ is equal to 0 in the same way. Specifically, if index $m$ is odd/even, and index $n$ is odd/even, $k_{2}$ equals to 0 in the expression of both Equations (11) and (13). As a result, odd side-band harmonics are eliminated in the output waveform and voltage of arm inductors around the odd carrier frequency multiples and the even side-band harmonics are eliminated in the output waveform and voltage of arm inductors around the even carrier-frequency multiples.

Harmonic-cancellation characteristics of $k_{1}, k_{3}$ and $k_{4}$ are affected by $\theta_{1}$ and $\theta_{2}$. Item $k_{1}$ can eliminate the harmonics of the whole group $m \omega_{c}$ with proper $\theta_{1}$, and the relationship between $k_{1}$ and $\theta_{1}$ is shown in Figure 4. If $\theta_{1}$ equals to $\pi / N$, all the even multiples carrier-frequency harmonic groups except those at $2 N$-multiples and its integral multiple are eliminated. For example, the harmonics of group $2 \omega_{c}$ and $4 \omega_{c}$ are eliminated when $N=3$. The harmonics of group $2 \omega_{c}, 4 \omega_{c}$ and $6 \omega_{c}$ are eliminated when $N=4$. If $\theta_{1}$ equals to $2 \pi / N$, all multiples carrier-frequency harmonic groups except those at $N$-multiples and its integral multiples are eliminated. For example, the harmonics of group $\omega_{c}, 2 \omega_{c}, 4 \omega_{c}$ and $5 \omega_{c}$ are eliminated when $N=3$. The harmonics of group $\omega_{c}, 2 \omega_{c}, 3 \omega_{c}, 5 \omega_{c}, 6 \omega_{c}$ and $7 \omega_{c}$ are eliminated when $N=4$.

After eliminating the harmonics of some whole group $m \omega_{c}$ with proper $\theta_{1}$, proper displacement angle $\theta_{2}$ can be selected to eliminate more harmonic components. The harmonic components of circulating current are the same as the voltage across the arm inductor. However, according to the relationship of trigonometric functions, $k_{3}$ in (12) and $k_{4}$ in (14) are completely different. For a particular harmonic group $m \omega_{c}$, output-voltage harmonics and the circulating current harmonic vary with $\theta_{2}$ at the completely opposite tendency. Displacement angle $\theta_{2}$ should be selected to obtain a zero $k_{3}$ or $k_{4}$ item coordinate with the harmonic-cancellation capability of $k_{2}$ to achieve maximal harmonic elimination of output voltage or circulating current, respectively. 


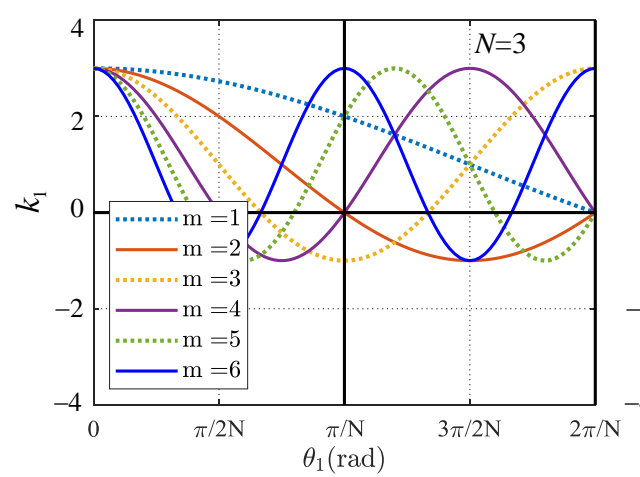

(a)

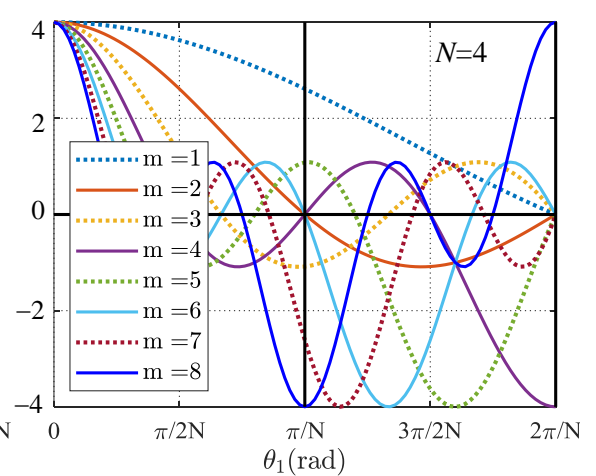

(b)

Figure 4. Harmonics of group cancellation characteristics with PSC-SPWM schemes. (a) $N=3$. (b) $N=4$.

For MMC, output voltage with lower harmonic distortion means smaller and lower-cost filters, and better output characteristics, especially when $N$ is small (e.g., in motor drive applications). On the other hand, the harmonics of circulating current causes higher loss and current stress. For a great number of SM applications (e.g., HVDC application), the harmonics of circulating current become the main problem. In this paper, five potential PSC-SPWM schemes (PSC1 to PSC5) for MMC that achieve maximal voltage harmonic elimination or maximal circulating current harmonic elimination are presented, as shown in Figure 5. They are described with a modulation wave frequency of $50 \mathrm{~Hz}$, a carrier frequency of $200 \mathrm{~Hz}$, and four SMs in each arm. Displacement angle $\theta_{1}, \theta_{2}$ of each scheme is given as follows.

PSC1:

$$
\left\{\begin{array}{l}
\theta_{1}=\frac{2 \pi}{N} \\
\theta_{2}=\frac{\pi}{N}+\pi
\end{array}\right.
$$

PSC2:

$$
\left\{\begin{array}{l}
\theta_{1}=\frac{2 \pi}{N} \\
\theta_{2}=\frac{\pi}{N}
\end{array}(N \text { is even }),\left\{\begin{array}{l}
\theta_{1}=\frac{2 \pi}{N} \\
\theta_{2}=0
\end{array}(N \text { is odd })\right.\right.
$$

PSC3:

$$
\left\{\begin{array}{l}
\theta_{1}=\frac{\pi}{N} \\
\theta_{2}=0
\end{array}\right.
$$

PSC4:

$$
\left\{\begin{array}{l}
\theta_{1}=\forall \theta \in(0,2 \pi / N] \\
\theta_{2}=\pi
\end{array}\right.
$$

PSC5:

$$
\left\{\begin{array}{l}
\theta_{1}=\frac{2 \pi}{N} \\
\theta_{2}=0
\end{array} \quad(N \text { is even }),\left\{\begin{array}{l}
\theta_{1}=\frac{2 \pi}{N} \\
\theta_{2}=\frac{\pi}{N}
\end{array} \quad(N \text { is odd })\right.\right.
$$

where PSC2 and PSC5 were mentioned in [22]. PSC1 and PSC3 were first proposed in [23], and PSC4 was added into this paper for discussion. Theoretically, PSC1, PSC2, and PSC 3 can achieve maximal voltage harmonic elimination, and PSC4 and PSC5 can achieve maximal circulating current harmonic elimination. Compared with PSC2 and PSC5, the proposed PSC1,PSC3, and PSC4 unify two different cases of odd and even SM situations. 

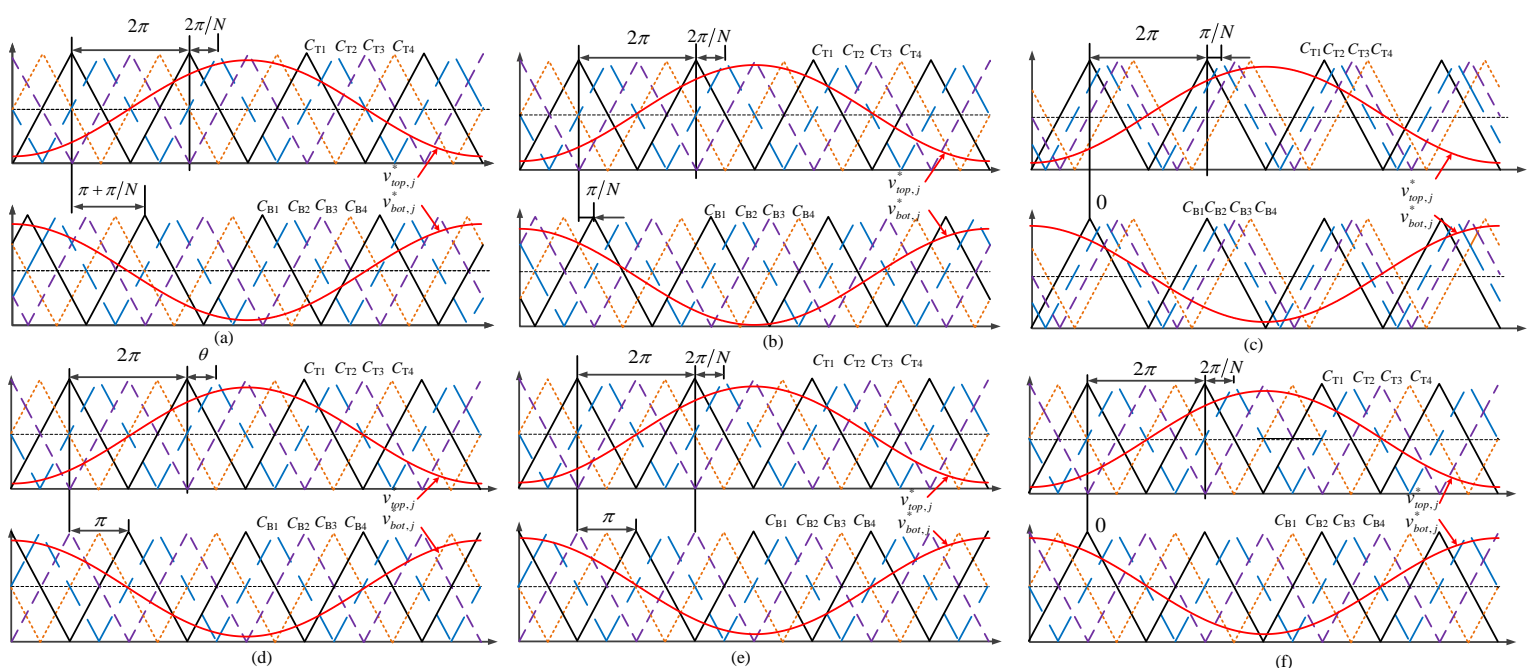

Figure 5. Proposed PSC-SPWM schemes for MMC that achieve maximal voltage harmonic elimination or maximal circulating current harmonic elimination. (a) PSC-SPWM1. (b) PSC-SPWM2.

(c) PSC-SPWM3. (d) PSC-SPWM4. (e) PSC-SPWM4 when $\theta_{1}=2 \pi / N$. (f) PSC-SPWM5.

For a specific index $m$, if both even $n$ and odd $n$ can result in a zero $k_{2}$ or $k_{3}$ item, the corresponding harmonic groups are eliminated in output voltage. Similarly, if both even $n$ and odd $n$ can result in a zero $k_{2}$ or $k_{4}$ item, the corresponding harmonic groups are eliminated in circulating current. For PSC1 and PSC2, all harmonics except those even side-band harmonics around the $(2 l+1) N$-multiples carrier frequency and the odd side-band harmonics around the $(2 l) N$-multiples carrier frequency remain with $\theta_{1}=2 \pi / N$. When the $m$ summation index is equal to $(2 l+1) N, k_{2}$ and $k_{3}$ of PSC1 and PSC2, it can be represented as follows.

PSC1:

$$
\left\{\begin{array}{l}
k_{2}=\sin \frac{((2 l+1) N+n) \pi}{2} \\
k_{3}=\sin \frac{((2 l+1)(N+1)+n) \pi}{2}
\end{array}\right.
$$

In (20), both even $n$ and odd $n$ can result in an even $((2 l+1) N+n)$ or even $((2 l+1)(N+1)+n)$, which obtains a zero $k_{2}$ or $k_{3}$ item. In other words, the harmonics of $(2 l+1) N$-multiples carrier frequency are totally eliminated in output voltage with PSC1, resulting in maximal voltage harmonic elimination.

PSC2:

$$
\left\{\begin{array}{l}
k_{2}=\sin \frac{((2 l+1) N+n) \pi}{2} \\
k_{3}=\sin \frac{(2 l+1+n) \pi}{2} \\
k_{2}=\sin \frac{((2 l+1) N+n) \pi}{2} \\
k_{3}=\sin \frac{n \pi}{2}
\end{array} \quad(N \text { is odd })\right.
$$

In (21), both even $n$ and odd $n$ can result in an even $((2 l+1) N+n)$ or $((2 l+1+n)$ when $N$ is even, and an even $((2 l+1) N+n)$ or even $n$ when $N$ is odd, which obtains a zero $k_{2}$ or $k_{3}$ item. As a result, the harmonics of $(2 l+1) N$-multiples carrier frequency are totally eliminated in output voltage with PSC2 the same with PSC1.

On the other hand, when the $m$ summation index is equal to $(2 l) N$, the $k_{2}$ and $k_{4}$ of PSC1 and PSC2 can be represented as 
PSC1:

$$
\left\{\begin{array}{l}
k_{2}=\sin \frac{(2 l N+n) \pi}{2} \\
k_{4}=\cos \frac{(2 l(N+1)+n) \pi}{2}
\end{array}\right.
$$

PSC2:

$$
\left\{\begin{array}{l}
k_{2}=\sin \frac{(2 l N+n) \pi}{2} \\
k_{4}=\cos \frac{(2 l+n) \pi}{2} \\
k_{2}=\sin \frac{(2 l N+n) \pi}{n \pi} \\
k_{4}=\cos \frac{n \pi}{2}
\end{array} \quad(N \text { is even })\right.
$$

In (22), both even $n$ and odd $n$ can result in an even $(2 l N+n)$ or odd $(2 l(N+1)+n)$, which obtains a zero $k_{2}$ or $k_{4}$ item. Similarly, in (23), both even $n$ and odd $n$ can result in an even $(2 l N+n)$ or odd $(2 l+n)$ when $N$ is even, and an even $(2 l N+n)$ or odd $n$ when $N$ is odd, which obtains a zero $k_{2}$ or $k_{4}$ item too. As a result, the extra harmonics of group $(2 l) N$-multiples carrier frequency are eliminated in circulating current with PSC1 or PSC2.

For PSC3, all harmonics of odd multiples and $(2 l) N$-multiples carrier frequency are remained when $\theta_{1}=\pi / N$. When the $m$ summation index is equal to $(2 l+1), k_{2}$ and $k_{3}$ can be represented as

PSC3:

$$
\left\{\begin{array}{l}
k_{2}=\sin \frac{(2 l+1+n) \pi}{2} \\
k_{3}=\sin \frac{n \pi}{2}
\end{array}\right.
$$

In (24), both even $n$ and odd $n$ can result in a zero $k_{2}$ or $k_{3}$ item. As a result, all odd multiples carrier-frequency harmonic groups are eliminated in output voltage with PSC3, resulting in maximal voltage harmonic elimination, the same as in PSC1 and PSC2.

On the other hand, when the $m$ summation index is equal to $(2 l) N$, the $k_{2}$ and $k_{4}$ of PSC 3 can be represented as

PSC3:

$$
\left\{\begin{array}{l}
k_{2}=\sin \frac{(2 l N+n) \pi}{2} \\
k_{4}=\cos \frac{n \pi}{2}
\end{array}\right.
$$

Both odd $n$ and even $n$ can result in a zero $k_{2}$ or $k_{4}$. The extra harmonics of group $(2 l) N$-multiples carrier frequency are eliminated in circulating current with PSC3.

For PSC4, $k_{2}$ and $k_{4}$ can be represented as

PSC4:

$$
\left\{\begin{array}{l}
k_{2}=\sin \frac{(m+n) \pi}{2} \\
k_{4}=\cos \frac{(m+n) \pi}{2}
\end{array}\right.
$$

All multiples carrier-frequency harmonic groups are eliminated in the circulating current with PSC4, while $k_{2}$ and $k_{3}$ can be represented as

PSC4:

$$
\left\{\begin{array}{l}
k_{2}=\sin \frac{(m+n) \pi}{2} \\
k_{3}=\sin \frac{(m+n) \pi}{2}
\end{array}\right.
$$

Only even side-band harmonics around the even multiples and the odd side-band harmonics are around the odd multiples are eliminated in the output voltage with PSC4 when $\theta_{1} \neq \pi / N$ and $\theta_{1} \neq 2 \pi / N$. 
For PSC5, when the $m$ summation index is equal to $l N, k_{2}$ and $k_{4}$ can be represented as PSC5:

$$
\left\{\begin{array}{l}
k_{2}=\sin \frac{(l N+n) \pi}{2} \\
k_{4}=\cos \frac{(l N+n) \pi}{2} \\
k_{2}=\sin \frac{(l N+n) \pi}{n \pi} \\
k_{4}=\cos \frac{n \pi}{2}
\end{array} \quad(N \text { is even })\right.
$$

All the harmonics of multiples carrier frequency are eliminated in the circulating current with a zero item $k_{2}$ or $k_{4}$ when PSC5 is applied.

On the other hand, when the $m$ summation index is equal to $l N, k_{2}$ and $k_{3}$ can be represented as PSC5:

$$
\begin{aligned}
& \left\{\begin{array}{l}
k_{2}=\sin \frac{(l N+n) \pi}{2} \\
k_{3}=\sin \frac{(l N+n) \pi}{2}
\end{array} \quad(N \text { is odd }),\right. \\
& k_{2}=\sin \frac{(l N+n) \pi}{2} \\
& k_{3}=\sin \frac{n \pi}{2}
\end{aligned} \quad(N \text { is even })
$$

There are no extra harmonics that can be eliminated in output voltage with PSC5.

From the above analysis, the conclusion is that only odd side-band harmonics around (2l) N-multiples carrier frequency are retained in output voltage with PSC1, PSC2, and PSC3 applied. The extra harmonic group of $(2 l) \mathrm{N}$-multiples carrier frequency is eliminated in circulating current with PSC1 and PSC2, while all even side-band harmonics around odd multiples carrier frequency remain with PSC3. All carrier-frequency harmonics are eliminated in the circulating current with both PSC4 and PSC5 applied. Simultaneously, maximal extra harmonic cancellation in output voltage can be obtained with PSC4 when $\theta_{1}=2 \pi / N$, which is equivalent to PSC5.

Carrier angles of each SM for five PSC-SPWM schemes when $N=3$ (odd number SMs example) and $N=4$ (even number SMs example) are listed in Figures 6 and 7. PSC1, PSC2, and PSC3 are the minimal voltage harmonic schemes. PSC4 (when $\theta_{1}=2 \pi / N$ ) and PSC5 are the minimal circulating current harmonic schemes. Considering that the status of each SM in the same arm is identical, PSC1 and PSC2, PSC4, and PSC5 are equivalent except that SMs in the bottom arm corresponding to the top arms are different. SMs with the same status (carrier angle) are filled with the same color and connected by red arrows. Obviously, the proposed PSC1 and PSC4 could automatically distribute the carrier angle.

\begin{tabular}{|cccccc}
\hline Angle & PSC1 & PSC2 & PSC3 & PSC4 & PSC5 \\
\hline$\theta_{\text {top }}(1)$ & $0^{\circ}$ & $0^{\circ}$ & $0^{\circ}$ & $0^{\circ}$ & $0^{\circ}$ \\
\hline$\theta_{\text {top }}(2)$ & $120^{\circ}$ & $120^{\circ}$ & $60^{\circ}$ & $120^{\circ}$ & $120^{\circ}$ \\
\hline$\theta_{\text {top }}(3)$ & $240^{\circ}$ & $240^{\circ}$ & $120^{\circ}$ & $240^{\circ}$ & $240^{\circ}$ \\
\hline$\theta_{b o t}(1)$ & $240^{\circ}$ & $0^{\circ}$ & $0^{\circ}$ & $180^{\circ}$ & $60^{\circ}$ \\
\hline$\theta_{b o t}(2)$ & $0^{\circ}$ & $120^{\circ}$ & $60^{\circ}$ & $300^{\circ}$ & $180^{\circ}$ \\
\hline$\theta_{b o t}(3)$ & $120^{\circ}$ & $240^{\circ}$ & $120^{\circ}$ & $60^{\circ}$ & $300^{\circ}$ \\
\hline
\end{tabular}

Figure 6. Carrier angles of each submodule (SM) for five PSC-SPWM schemes when $N=3$. 


\begin{tabular}{|ccccccc}
\hline Angle & PSC1 & PSC2 & PSC3 & PSC4 & PSC5 \\
\hline$\theta_{\text {top }}(1)$ & $0^{\circ}$ & $0^{\circ}$ & $0^{\circ}$ & $0^{\circ}$ & $0^{\circ}$ \\
\hline$\theta_{\text {top }}(2)$ & $90^{\circ}$ & $90^{\circ}$ & $45^{\circ}$ & $90^{\circ}$ & $90^{\circ}$ \\
\hline$\theta_{\text {top }}(3)$ & $180^{\circ}$ & $180^{\circ}$ & $90^{\circ}$ & $180^{\circ}$ & $180^{\circ}$ \\
\hline$\theta_{\text {top }}(4)$ & $270^{\circ}$ & $270^{\circ}$ & $135^{\circ}$ & $270^{\circ}$ & $270^{\circ}$ \\
\hline$\theta_{b o t}(1)$ & $225^{\circ}$ & $45^{\circ}$ & $0^{\circ}$ & $180^{\circ}$ & $0^{\circ}$ \\
\hline$\theta_{b o t}(2)$ & $315^{\circ}$ & $135^{\circ}$ & $45^{\circ}$ & $270^{\circ}$ & $90^{\circ}$ \\
\hline$\theta_{b o t}(3)$ & $45^{\circ}$ & $225^{\circ}$ & $90^{\circ}$ & $0^{\circ}$ & $180^{\circ}$ \\
\hline$\theta_{b o t}(4)$ & $135^{\circ}$ & $-315^{\circ}$ & $135^{\circ}$ & $90^{\circ}$ & $270^{\circ}$ \\
\hline
\end{tabular}

Figure 7. Carrier angles of each SM for five PSC-SPWM schemes when $N=4$.

The specific PSC-SPWM scheme is determined according to the number of SMs and aforementioned harmonic-cancellation characteristics analysis. Considering the harmonic performance of both output voltage and circulating current, the optimal maximal output voltage and circulating current harmonic elimination schemes are PSC1 and PSC4 when $\theta_{1}=2 \pi / N$, respectively, for which implementation does not need irregular change with parity of submodule number.

\subsection{Capacitor Voltage of SMs Affected by PSC-SPWM Schemes}

The above analysis is based on the assumption that all SM capacitor voltages are naturally balanced and voltage fluctuations are ignored. However, SM voltage is also affected by modulation methods. From (5), capacitor voltage varies with switching function, load current, and circulating current. The voltage fluctuation of SM capacitors in one carrier cycle is given as

$$
\left\{\begin{aligned}
\Delta v_{t o p, j}(k) & =\frac{1}{C_{\mathrm{SM}}} \int_{0}^{T_{S}}\left(S_{t o p, j}(k) i_{z, j}+0.5 S_{t o p, j}(k) i_{s, j}\right) d t \\
\Delta v_{b o t, j}(k) & =\frac{1}{C_{\mathrm{SM}}} \int_{0}^{T_{S}}\left(S_{b o t, j}(k) i_{z, j}-0.5 S_{b o t, j}(k) i_{s, j}\right) d t
\end{aligned}\right.
$$

where $T_{S}$ is a fundamental period. SM capacitor voltage is affected by switching function, circulating current and the load current. Natural balancing of the 2-Cell modular multilevel converter with traditional PSC-SPWM schemes was discussed in [24], which is closely related to harmonic distribution of output current and circulating current. If the switching function and circulating current contain the same harmonic components, or the switching function and phase current contain the same harmonic components in the orthogonal trigonometric system, the right side of (21) could contain a non-negligible DC component. Voltage variation in a fundamental cycle is not equal to zero, and the capacitor voltage of each SM deviates from the rated value.

According to the above analysis, even side-band harmonics around the carrier frequency are remained in circulating current with PSC3. However, these harmonic components are also contained in switching functions $S_{t o p, j}$ and $S_{b o t, j}$. In an orthogonal trigonometric function system, these large harmonic components could cause a non-negligible DC current in SM capacitors, which results in voltage deviation. The capacitor-voltage deviation phenomenon is verified by simulation and experiments in next section. Similarly, even side-band harmonics around the carrier frequency remain in output current with PSC4 when $\theta_{1}=\pi / N$, which also results in voltage deviation. Therefore, from the perspective of the effect on capacitor voltage, $\theta_{1}=2 \pi / N$ should be met for PSC-SPWM schemes.

\section{Simulation and Experiment Results}

Simulation and experiment results were obtained to verify the analysis of the PSC-SPWM strategy in a three-phase MMC with four half-bridge SMs. Simulation results were obtained from 
MATLAB/Simulink, and the experiment results were obtained from a low-power laboratory prototype as shown in Figure 8. A digital-signal processor (DSP; TI TMS32028335) was used to generate the reference voltage, while two field-programmable gate arrays (FPGAs; Spartan-6 XC6SLX9)were adopted to generate the triangular carriers and data processing, respectively. PWM signals were transmitted to SMs via optical fibers. The main parameters of the converter are given in Table 1.

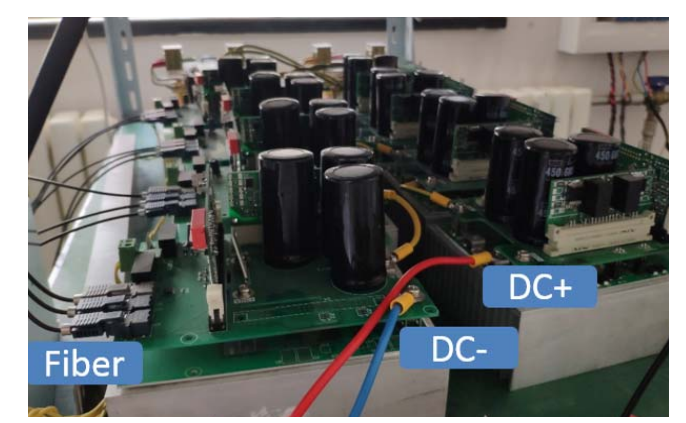

Figure 8. Laboratory prototype.

Table 1. MMC parameters.

\begin{tabular}{ccc}
\hline Quantity & Symbol & Values \\
\hline DC bus voltage & $v_{D C}$ & $200 \mathrm{~V}$ \\
Submodule capacitance & $C_{S m}$ & $3.6 \mathrm{mF}$ \\
Number of submodules & $N$ & 4 \\
Bridge inductance & $L_{\text {arm }}$ & $2 \mathrm{mH}$ \\
Carrier frequency & $f_{C}$ & $1000 \mathrm{~Hz}$ \\
Load inductance & $L$ & $5 \mathrm{mH}$ \\
Load resistance & $R$ & $24 \Omega$ \\
Modulation index & $M$ & 0.8 \\
\hline
\end{tabular}

\subsection{Simulation Results}

Figures 9-13 shows the simulation results of output-phase voltage with five PSC-SPWM strategies. The equivalent output-phase voltage reached $2 N+1$ levels with PSC1, PSC2, and PSC3, which had maximal output-voltage harmonic-cancellation ability, and voltage total harmonic distortion (THD) was $14.7 \%$ with the main odd side-band harmonics around $2 \mathrm{~N}$-multiples carrier frequency and its integral multiple harmonic groups. The simulation results of PSC4, when $\theta_{1}=2 \pi / N$, and PSC 5 are shown in Figures 12 and 13, respectively. Both of these PSC-SPWM schemes had the same output characteristics. Equivalent output voltage reached $N+1$ levels, and voltage THD was much larger than that of PSC1, PSC2, and PSC3, with $36.223 \%$. The main harmonic components were around $N$ times the carrier frequency and its integral multiples.

Simulation results of circulating current under five PSC-SPWM strategies are shown in Figures 14-18. The circulating current included the extra $N$-multiples carrier frequency and its odd multiple harmonic groups with PSC1 and PSC2 applied, all odd carrier multiple harmonic groups remained with PSC3 applied. The circulating current was mainly composed of the DC component and the secondary component with PSC 4 when $\theta_{1}=2 \pi / N$ and PSC5. All high-frequency harmonics were completely eliminated, with maximal harmonic-cancellation ability. On the other hand, the harmonic characteristic of output-phase voltage was decreased. Crculating current harmonic and output-voltage harmonics varied with $\theta_{2}$ at a completely opposite tendency. 


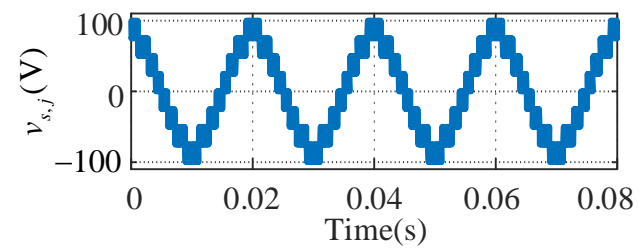

(a)

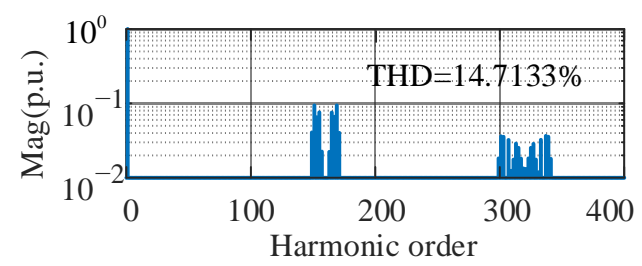

(b)

Figure 9. Theoretical output voltage of PSC1. (a) Waveform of equivalent output-phase voltage; (b) fast Fourier transform (FFT) analysis.

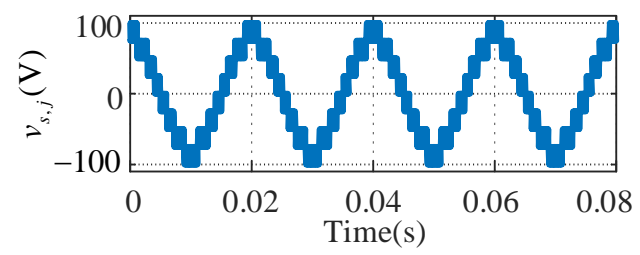

(a)

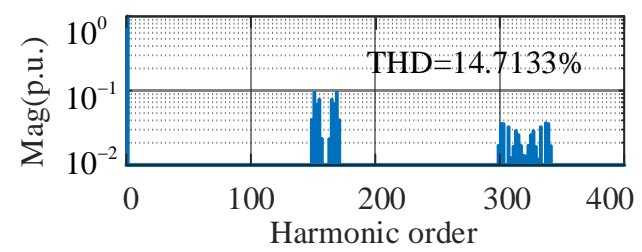

(b)

Figure 10. Theoretical output voltage of PSC2. (a) Waveform of equivalent output-phase voltage; (b) FFT analysis.

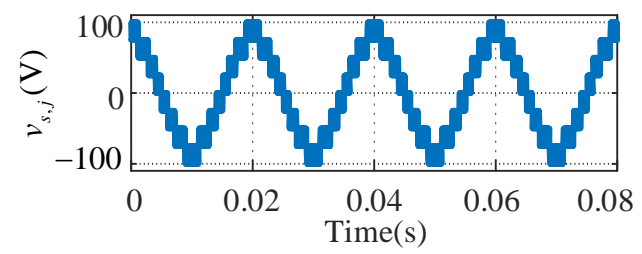

(a)

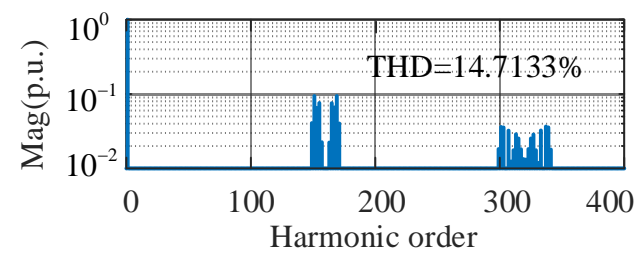

(b)

Figure 11. Theoretical output voltage of PSC3. (a) Waveform of equivalent output-phase voltage; (b) FFT analysis. 


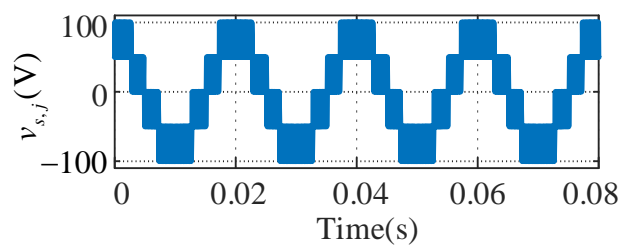

(a)

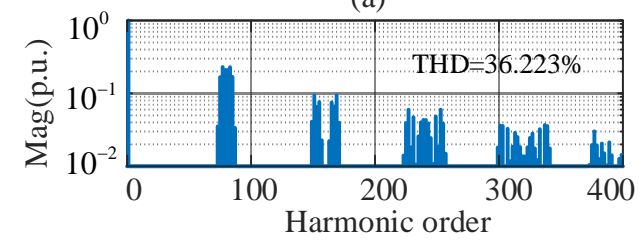

(b)

Figure 12. Theoretical output voltage of PSC4. (a) Waveform of equivalent output-phase voltage; (b) FFT analysis.
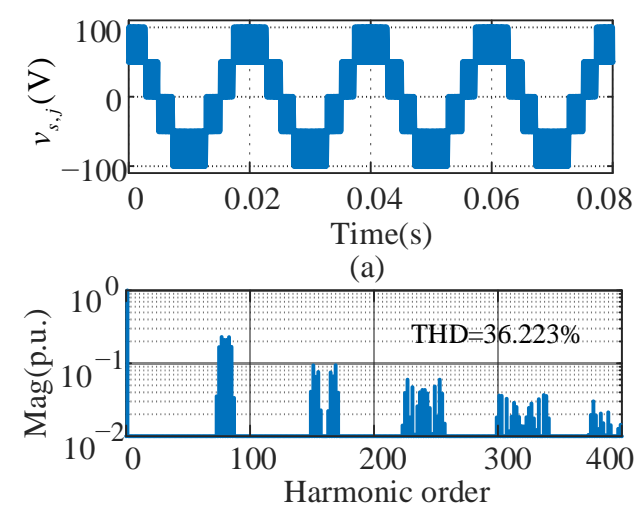

(b)

Figure 13. Theoretical output voltage of PSC5. (a) Waveform of equivalent output-phase voltage; (b) FFT analysis.

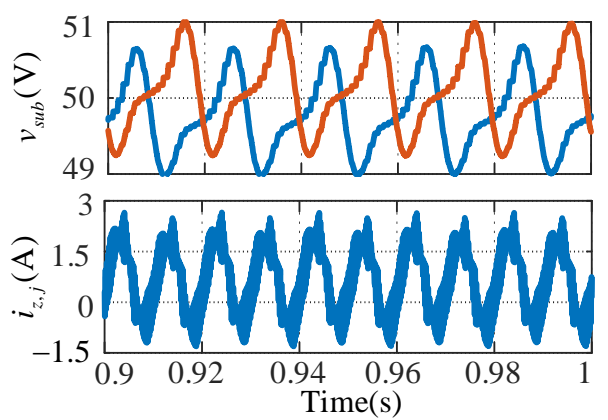

(a)

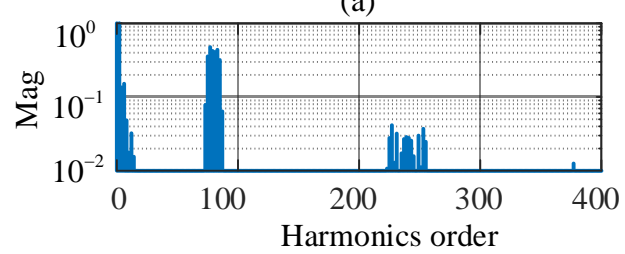

(b)

Figure 14. Simulation results of PSC1. (a) Capacitor voltage of SMs and circulating current. (b) FFT analysis of circulating current. 

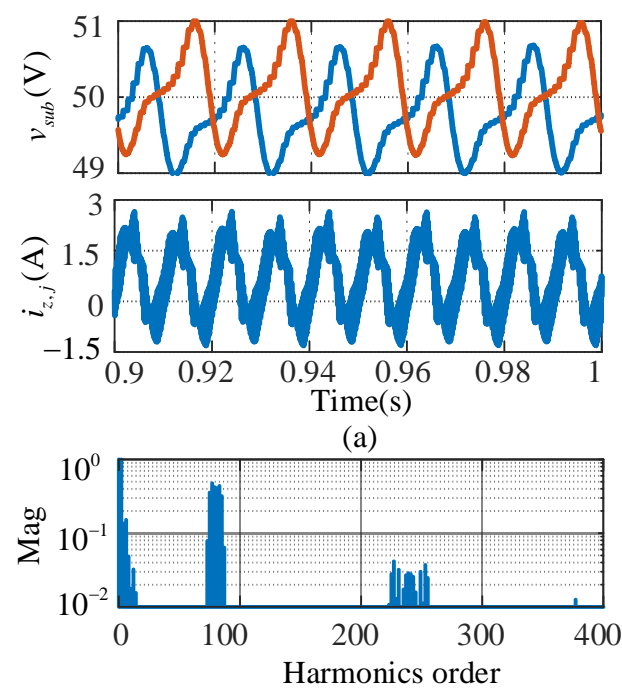

(b)

Figure 15. Simulation results of PSC2. (a) Capacitor voltage of SMs and circulating current. (b) FFT analysis of circulating current.

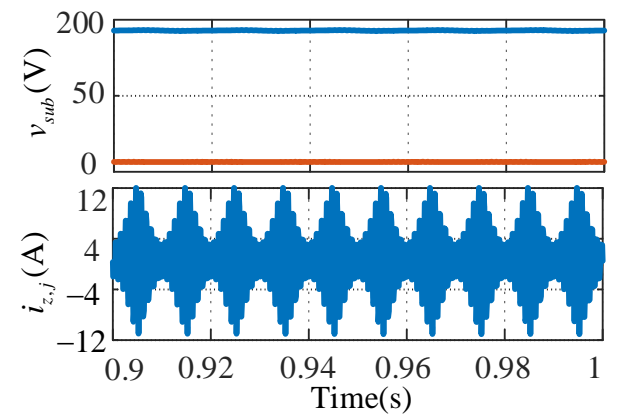

(a)

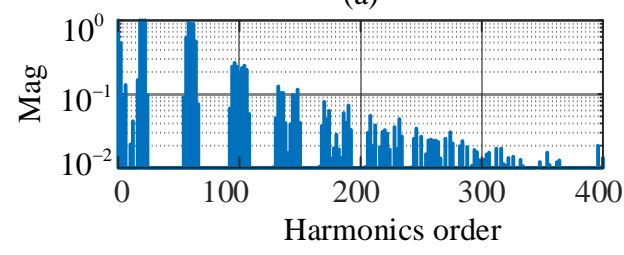

(b)

Figure 16. Simulation results of PSC3. (a) Capacitor voltage of SMs and circulating current. (b) FFT analysis of circulating current.

As shown in Figure 16, PSC3 caused the deviation and unbalance of capacitor voltage because large even side-band harmonics around the carrier frequency (especially h18, h20, and h22) remained in circulating current with PSC3, which were also contained in switching function of SMs. These harmonic components caused a non-negligible DC current in the capacitor of the SMs. This phenomenon can also be intuitively understood from the number of submodules connected to each phase. Unlike other solutions, 0 to $2 \mathrm{~N}$ SMs could be put into a phase with PSC3. From (1), when $2 N$ SMs were inserted, voltage across the arm inductors was $v_{D C}$, causing high circulating current and unstable SM capacitor voltages. Therefore, the selection of $\theta_{1}$ and $\theta_{2}$ needs to consider the harmonic-cancellation characteristic of both output voltage and circulating current. PSC1 and PSC4 when $\theta_{1}=2 \pi / N$ are the optimal output-voltage harmonic minimization PSC-SPWM scheme and the optimal circulating current harmonic minimization PSC-SPWM scheme, respectively, with a stable and balanced capacitor voltage. 


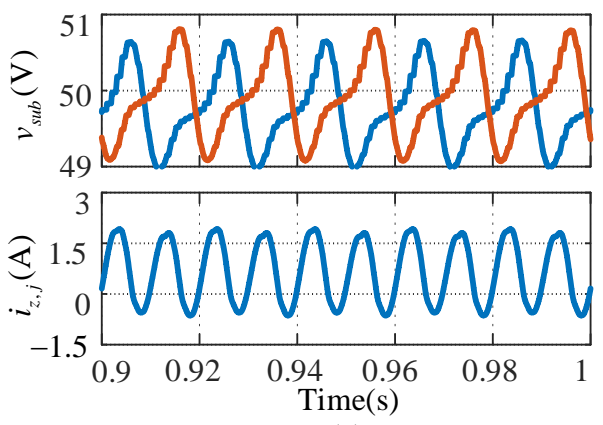

(a)

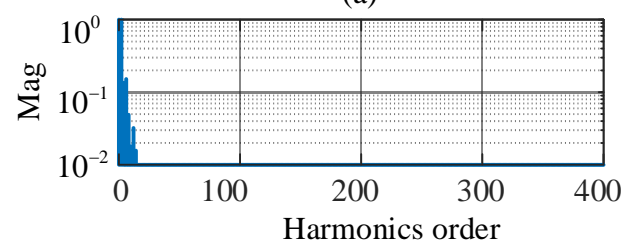

(b)

Figure 17. Simulation results of PSC4. (a) Capacitor voltage of SMs and circulating current. (b) FFT analysis of circulating current.

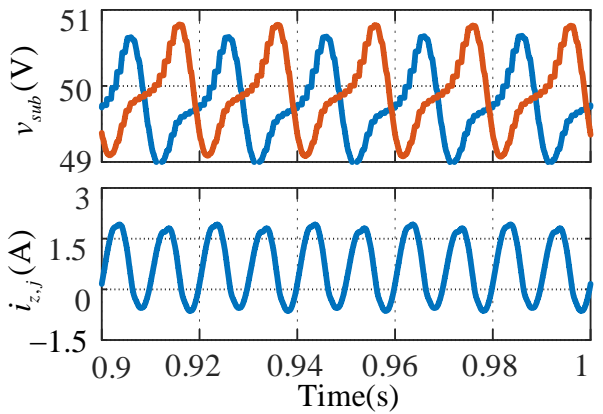

(a)

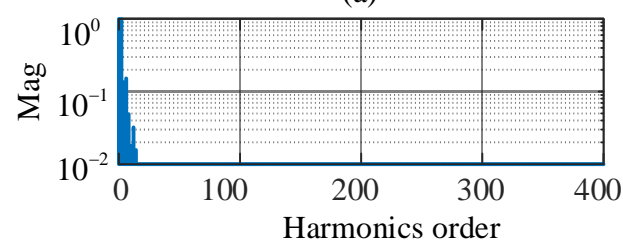

(b)

Figure 18. Simulation results of PSC5. (a) Capacitor voltage of SMs and circulating current. (b) FFT analysis of circulating current.

\subsection{Experiment Results}

Figure 19 shows the experiment results with PSC1, where displacement angles were selected as $\theta_{1}=90^{\circ}$ and $\theta_{2}=225^{\circ}$. Experiment results were almost identical to the simulation results, including output-voltage levels, the harmonic distribution of output voltage and circulating current, and the voltage stability of capacitors; $2 N+1$ voltage levels were generated, and output THD was $13.75 \%$, which was close to the theoretical $14.71 \%$. Simultaneously, high-frequency harmonic groups (mainly side-band harmonics around $(2 l+1) \mathrm{N}$-multiples carrier frequency) remained in the circulating current, which was calculated by a mathematical function of an oscilloscope according to (2). The peak value of circulating current was approximately equal to $1.5 \mathrm{~A}$ capacitor voltage of SMs in the top and bottom arms, which were naturally balanced with small fluctuation.

Figure 20 shows the experiment results with PSC2, where displacement angles were selected as $\theta_{1}=90^{\circ}$ and $\theta_{2}=45^{\circ}$. PSC2 achieved the same characteristics as those of PSC1. The equivalence of PSC1 and PSC2 was verified as analyzed before, and only SMs corresponding to the top and bottom 
arms were different; the proposed PSC1 unified two different cases of odd and even SM situations for maximal harmonic elimination of output voltage.

Figure 21 shows the experiment results with PSC3, where displacement angles were selected as $\theta_{1}=45^{\circ}$ and $\theta_{2}=0^{\circ}$. Only three levels of output voltage were generated. Theoretical $2 \mathrm{~N}+1$ output-voltage levels could not be achieved because the capacitor voltage of SMs deviated to $v_{D C}$ or 0 , which was consistent with the simulation results. This is a validation that PSC 3 is not acceptable for industrial applications, and $\theta_{1}=2 \pi / N$ should be guaranteed for PSC-SPWM schemes.

Figure 22 shows the experiment results with PSC4, where displacement angles were selected as $\theta_{1}=90^{\circ}$ and $\theta_{2}=180^{\circ}$. Experiment results were almost identical to the simulation results; $N+1$ voltage levels were generated, and output THD was $32.42 \%$, which was close to the theoretical $36.23 \%$. Simultaneously, all high-frequency harmonic groups were completely eliminated in the circulating current, and the peak value of the circulating current was approximately equal to $0.5 \mathrm{~A}$, which was smaller than that with PSC1 and PSC2. Capacitor voltages of SMs in the top and bottom arms were naturally balanced with small fluctuation. PSC 4 when $\theta_{1} \neq 2 \pi / N$ caused the deviation and unbalance of capacitor voltage because of the same reason as that for PSC3.

Figure 23 shows the experiment results with PSC5, where displacement angles were selected as $\theta_{1}=90^{\circ}$ and $\theta_{2}=0^{\circ}$. PSC5 achieved the same characteristics as those of PSC4. PSC 4 and PSC5 were equivalent as analyzed before, and only SMs corresponding to the top and bottom arms were different. The proposed PSC 4 when $\theta_{1}=2 \pi / N$ could unify two different cases of odd and even SM situations for the maximal harmonic elimination of the circulating current.

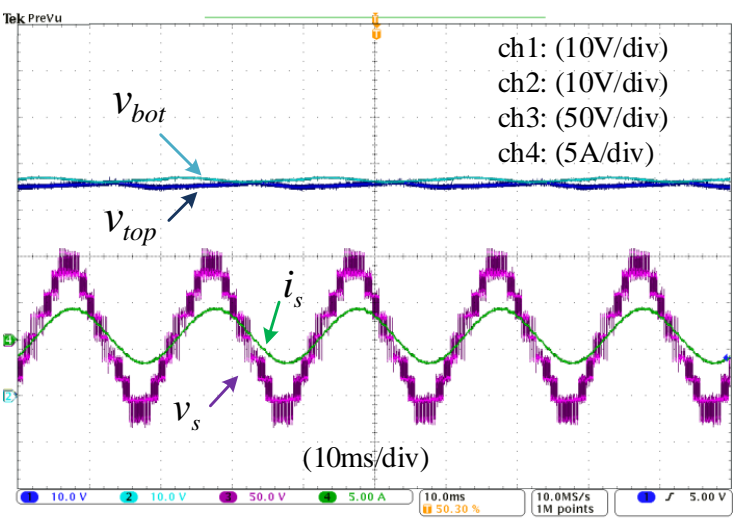

(a)

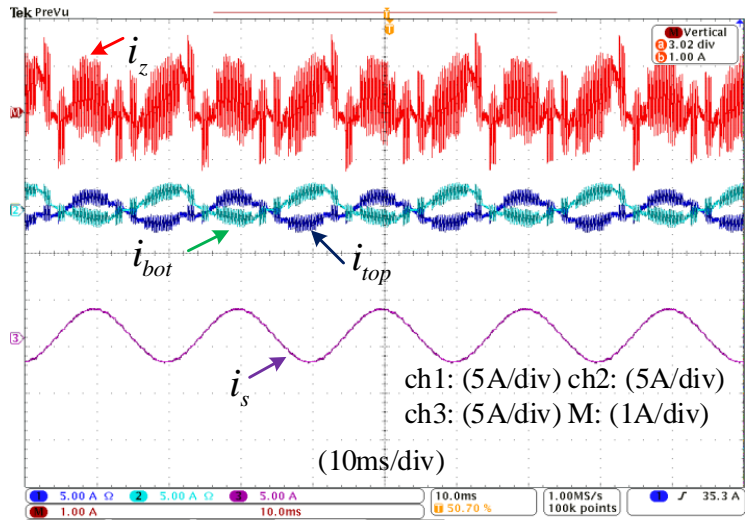

(b)

Figure 19. Experiment results with PSC1. (a) Phase voltage $v_{S}$, phase current $i_{s}$, capacitor voltage of $\mathrm{SM} v_{\text {top }}$ and $v_{\text {bot }} ;(\mathbf{b})$ circulating current $i_{z}$, phase current $i_{s}$, arm current $i_{\text {top }}$, and $i_{\text {bot }}$.

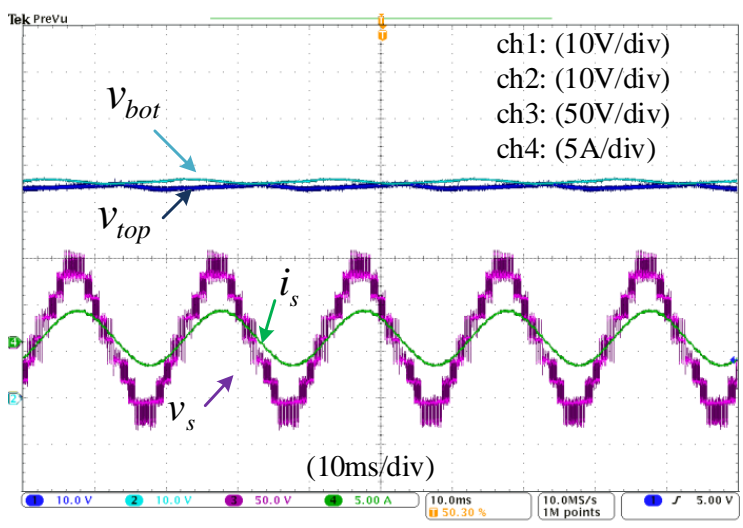

(a)

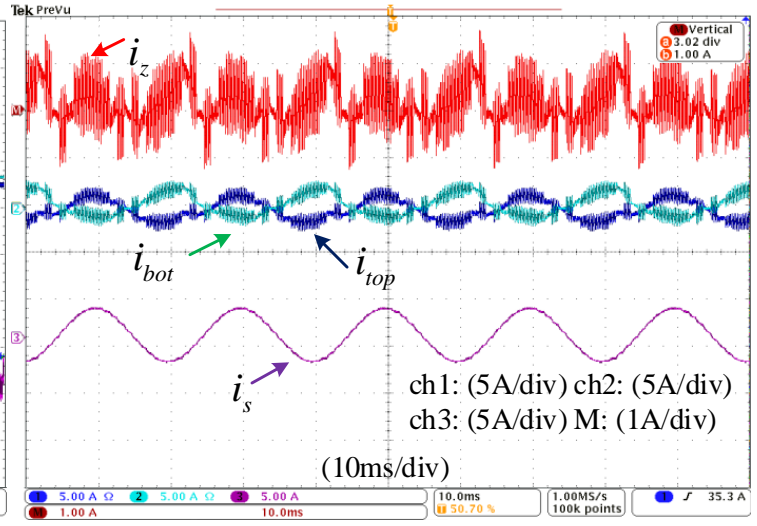

(b)

Figure 20. Experiment results with PSC2. (a) Phase voltage $v_{s}$, phase current $i_{s}$, capacitor voltage of $\mathrm{SM} v_{\text {top }}$ and $v_{\text {bot }} ;(\mathbf{b})$ circulating current $i_{z}$, phase current $i_{s}$, arm current $i_{\text {top }}$ and $i_{\text {bot }}$. 


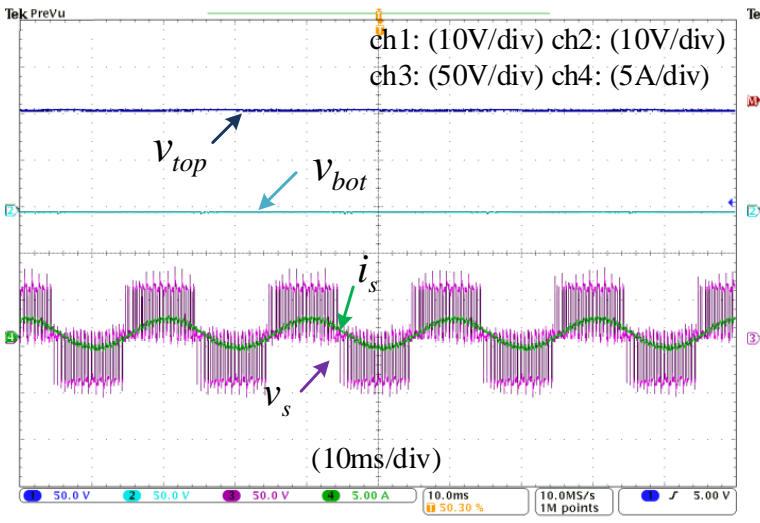

(a)

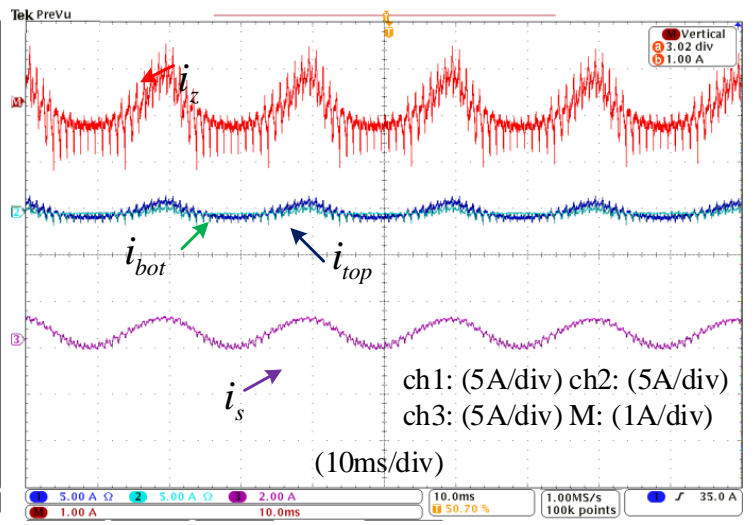

(b)

Figure 21. Experiment results with PSC3. (a) Phase voltage $v_{s}$, phase current $i_{s}$, capacitor voltage of $\mathrm{SM} v_{\text {top }}$ and $v_{\text {bot }} ;(\mathbf{b})$ circulating current $i_{z}$, phase current $i_{s}$, arm current $i_{\text {top }}$ and $i_{\text {bot }}$.

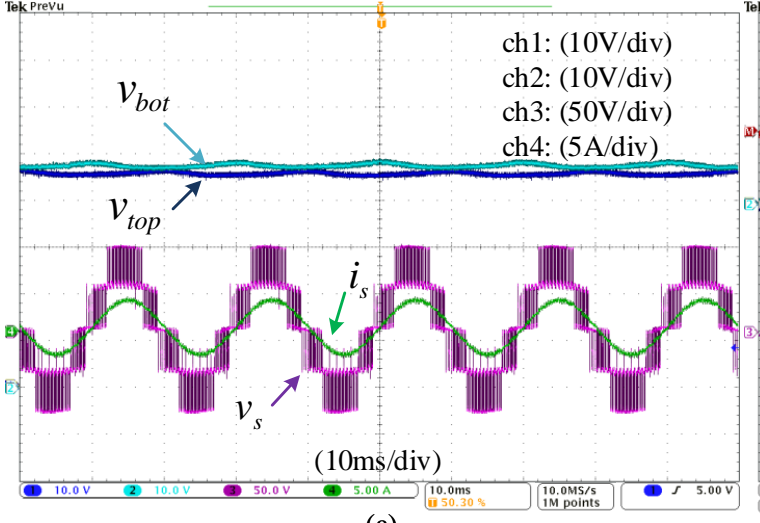

(a)

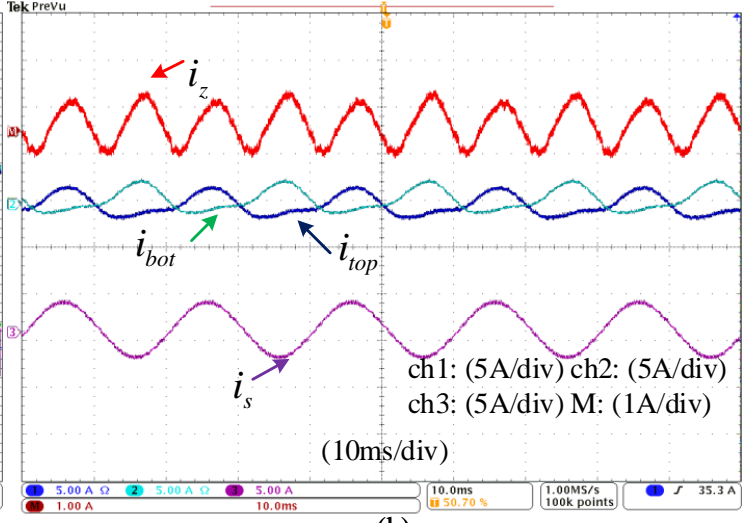

(b)

Figure 22. Experiment results with PSC 4 when $\theta_{1}=2 \pi / N$. (a) Phase voltage $v_{s}$, phase current $i_{S}$, capacitor voltage of SM $v_{\text {top }}$ and $v_{\text {bot }} ;(\mathbf{b})$ circulating current $i_{z}$, phase current $i_{S}$, arm current $i_{\text {top }}$ and $i_{b o t}$.

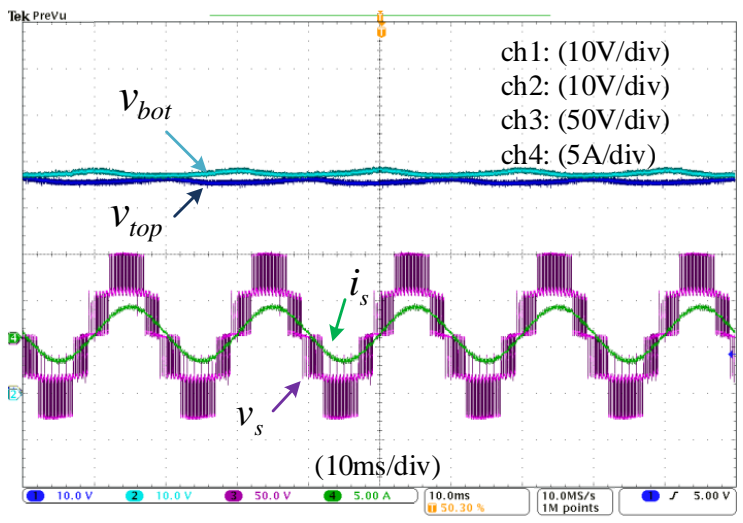

(a)

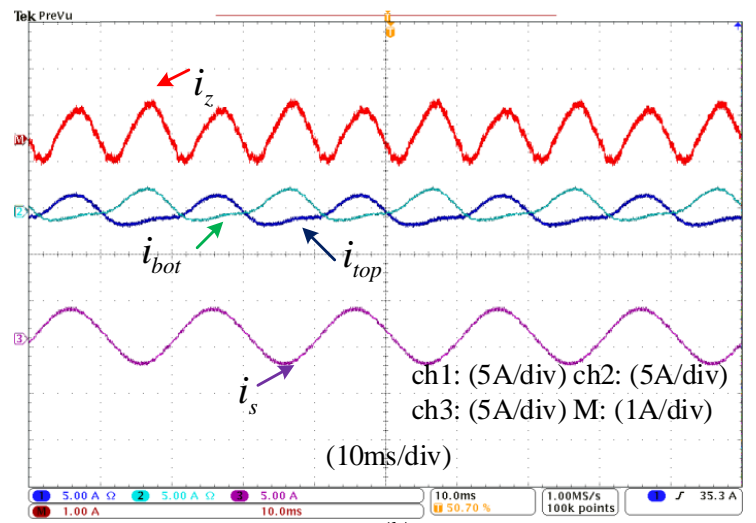

(b)

Figure 23. Experiment results with PSC5. (a) Phase voltage $v_{s}$, phase current $i_{s}$, capacitor voltage of SMs $v_{\text {top }}$ and $v_{\text {bot }} ;(\mathbf{b})$ circulating current $i_{z}$, phase current $i_{s}$, arm current $i_{\text {top }}$ and $i_{\text {bot }}$.

The PSC-SPWM of MMC is different from that of CHB, and only those SMs in the identical arm are equivalent. In order to avoid deviation of capacitor voltage, the selection of $\theta_{1}$ and $\theta_{2}$ needs to consider harmonic cancellation characteristics of both output voltage and circulating current. A comparison of five PSC-SPWM schemes is listed in Table 2. Considering these facts and implementations, PSC1 
$\left(\theta_{1}=2 \pi / N, \theta_{2}=\pi+\pi / N\right)$ is the most suitable PSC-SPWM scheme in practical applications when the number of SMs is small. Only odd side-band harmonics around $(2 l) N$-multiples carrier frequency remained in output voltage, and even side-band harmonics around $(2 l-1) N$-multiples carrier frequency remained in circulating current; at the same time, the capacitor voltage of SMs was stable and balanced. On the other hand, PSC4 $\left(\theta_{1}=2 \pi / N, \theta_{2}=\pi\right)$ is the most suitable PSC-SPWM scheme in practical applications when the number of SMs is large. Only side-band harmonics around (l) $\mathrm{N}$-multiples carrier frequency remained in output voltage, and all carrier-frequency harmonics were eliminated in circulating current; at the same time, the capacitor voltage of SMs was stable and balanced. Moreover, the implementations of both PSC1 and PSC 4 when $\theta_{1}=2 \pi / N$ do not need irregular change with the parity of submodule number.

Table 2. Comparison of five PSC-SPWM schemes.

\begin{tabular}{llllll}
\hline & PSC1 & PSC2 & PSC3 & PSC4 & PSC5 \\
\hline Output level & $2 N+1$ & $2 N+1$ & $2 N+1$ & $N+1$ & $N+1$ \\
\hline Output-voltage harmonics & $2 l N f_{c}$ & $2 l N f_{c}$ & $2 l N f_{c}$ & $l N f_{c}$ & $l N f_{c}$ \\
\hline Circulating-current harmonics & $(2 l-1) N f_{c}$ & $(2 l-1) N f_{c}$ & $(2 l-1) f_{c}$ & 0 & 0 \\
\hline Expression of displacement angles & fixed & Different with parity of $N$ & fixed & fixed & Different with parity of $N$ \\
\hline Voltage stability of capacitors & stable & stable & unstable & stable & stable \\
\hline
\end{tabular}

\section{Conclusions}

This paper provided theoretical and experimental discussions on the characteristics of the modular multilevel converter (MMC) when phase-shifted carrier sinusoidal pulse width modulation (PSC-SPWM) is applied. The harmonic components of output phase voltage and circulating current were derived in detail. We also analyzed how the displacement angle between SM carriers affects the harmonic-cancellation characteristics of output voltage, circulating current, and capacitor voltage of SMs. Displacement angles were found to affect the harmonic magnitudes of both output voltage and circulating current. On the basis of analysis, five potential PSC-SPWM schemes with maximal harmonic-cancellation ability for output voltage or circulating current were proposed. Furthermore, capacitor voltages affected by PSC-SPWM schemes were analyzed. Optimal displacement angles were selected for minimizing the harmonics of output voltage and circulating current that are suitable for application with a low and high number of SMs. Lastly, the mathematical analysis and proposed schemes were verified by simulation and experiments on a low-power MMC prototype.

Author Contributions: Conceptualization, C.W. and Q.C.; methodology, Q.C.; software, Q.C. and J.W.; validation, Q.C.; formal analysis, Q.C.; investigation, Q.C.; resources, J.W.; data curation, Q.C.; writing-original-draft preparation, Q.C.; writing - review and editing, Q.C., C.W. and J.W.; visualization, Q.C.; supervision, C.W.; project administration, C.W.; funding acquisition, C.W. All authors have read and agreed to the published version of the manuscript.

Funding: This research received no external funding.

Acknowledgments: We thank all the journal editors and the reviewers for their valuable time and constructive comments that have contributed to improving this manuscript.

Conflicts of Interest: The authors declare no conflict of interest.

\section{References}

1. Lesnicar, A.; Marquardt, R. An innovative modular multilevel converter suitable for a wide power range. In Proceedings of the 2003 IEEE Bologna Power Tech Conference Proceedings, Bologna, Italy, 23-26 June 2003; Volume 3, p. 6.

2. Dekka, A.; Wu, B.; Fuentes, R.L.; Perez, M.; Zargari, N.R. Evolution of Topologies, Modeling, Control Schemes, and Applications of Modular Multilevel Converters. IEEE Trans. Emerg. Sel. Topics Power Electron. 2017, 5, 1631-1656. [CrossRef] 
3. Zhang, J.; Xu, S.; Din, Z.; Hu, X. Hybrid Multilevel Converters: Topologies, Evolutions and Verifications. Energies 2019, 12, 615. [CrossRef]

4. Solas, E.; Abad, G.; Barrena, J.A.; Aurtenetxea, S.; Cárcar, A.; Zając, L. Modular Multilevel Converter With Different Submodule Concepts-Part II: Experimental Validation and Comparison for HVDC Application. IEEE Trans. Ind. Electron. 2013, 60, 4536-4545. [CrossRef]

5. Yang, X.; Xue, Y.; Chen, B.; Lin, Z.; Mu, Y.; Zheng, T.Q.; Igarashi, S.; Li, Y. An enhanced reverse blocking MMC with DC fault handling capability for HVDC applications. Electr. Power Syst. Res. 2018, 163, 706-714. [CrossRef]

6. Bina, M.T. A Transformerless Medium-Voltage STATCOM Topology Based on Extended Modular Multilevel Converters. IEEE Trans. Power Electron. 2011, 26, 1534-1545.

7. Cupertino, A.F.; Farias, J.V.M.; Pereira, H.A.; Seleme, S.I.; Teodorescu, R. Comparison of DSCC and SDBC Modular Multilevel Converters for STATCOM Application During Negative Sequence Compensation. IEEE Trans. Ind. Electron. 2019, 66, 2302-2312. [CrossRef]

8. Chen, Y.; Zhao, S.; Li, Z.; Wei, X.; Kang, Y. Modeling and Control of the Isolated DC-DC Modular Multilevel Converter for Electric Ship Medium Voltage Direct Current Power System. IEEE Trans. Emerg. Sel. Topics Power Electron. 2017, 5, 124-139. [CrossRef]

9. Hagiwara, M.; Nishimura, K.; Akagi, H. A Medium-Voltage Motor Drive With a Modular Multilevel PWM Inverter. IEEE Trans. Power Electron. 2010, 25, 1786-1799. [CrossRef]

10. Sau, S.; Fernandes, B.G. Modular Multilevel Converter Based Variable Speed Drive With Reduced Capacitor Ripple Voltage. IEEE Trans. Ind. Electron. 2019, 66, 3412-3421. [CrossRef]

11. Ma, F.; Xu, Q.; He, Z.; Tu, C.; Shuai, Z.; Luo, A.; Li, Y. A Railway Traction Power Conditioner Using Modular Multilevel Converter and Its Control Strategy for High-Speed Railway System. IEEE Trans. Transp. Electrif. 2016, 2, 96-109. [CrossRef]

12. Wang, Z.; Zheng, Z.; Li, Y.; Li, G. Modulation and Control Strategy for Electric Traction Drive System of Rail Transit Vehicles. Trans. China Electrotech. Soc. 2016, 31, 223-232.

13. Konstantinou, G.; Pou, J.; Ceballos, S.; Darus, R.; Agelidis, V.G. Switching Frequency Analysis of Staircase-Modulated Modular Multilevel Converters and Equivalent PWM Techniques. IEEE Trans. Power Del. 2016, 31, 28-36. [CrossRef]

14. Samajdar, D.; Bhattacharya, T.; Dey, S. A Reduced Switching Frequency Sorting Algorithm for Modular Multilevel Converter With Circulating Current Suppression Feature. IEEE Trans. Power Electron. 2019, 34, 10480-10491. [CrossRef]

15. Tu, Q.; Xu, Z.; Xu, L. Reduced Switching-Frequency Modulation and Circulating Current Suppression for Modular Multilevel Converters. IEEE Trans. Power Del. 2011, 26, 2009-2017.

16. Pérez-Basante, A.; Ceballos, S.; Konstantinou, G.; Pou, J.; Andreu, J.; de Alegría, I.M. (2N+1) Selective Harmonic Elimination-PWM for Modular Multilevel Converters: A Generalized Formulation and A Circulating Current Control Method. IEEE Trans. Power Electron. 2018, 33, 802-818. [CrossRef]

17. Dekka, A.; Wu, B.; Zargari, N.R.; Fuentes, R.L. A Space-Vector PWM-Based Voltage-Balancing Approach With Reduced Current Sensors for Modular Multilevel Converter. IEEE Trans. Ind. Electron. 2016, 63, 2734-2745. [CrossRef]

18. Ronanki, D.; Williamson, S.S. A Simplified Space Vector Pulse Width Modulation Implementation in Modular Multilevel Converters for Electric Ship Propulsion Systems. IEEE Trans. Transp. Electrif. 2019, 5, 335-342. [CrossRef]

19. Ilves, K.; Harnefors, L.; Norrga, S.; Nee, H. Analysis and Operation of Modular Multilevel Converters with Phase-Shifted Carrier PWM. IEEE Trans. Power Electron. 2015, 30, 268-283. [CrossRef]

20. Zhou, D.; Yang, S.; Tang, Y. Model-Predictive Current Control of Modular Multilevel Converters With Phase-Shifted Pulsewidth Modulation. IEEE Trans. Ind. Electron. 2019, 66, 4368-4378. [CrossRef]

21. Sasongko, F.; Sekiguchi, K.; Oguma, K.; Hagiwara, M.; Akagi, H. Theory and Experiment on an Optimal Carrier Frequency of a Modular Multilevel Cascade Converter With Phase-Shifted PWM. IEEE Trans. Power Electron. 2016, 31, 3456-3471. [CrossRef]

22. Li, B.; Yang, R.; Xu, D.; Wang, G.; Wang, W.; Xu, D. Analysis of the Phase-Shifted Carrier Modulation for Modular Multilevel Converters. IEEE Trans. Power Electron. 2015, 30, 297-310. [CrossRef] 
23. Cheng, Q.; Wang, C. Comparison of Phase-Shifted Carrier PWM Schemes for Modular Multilevel Converter. In Proceedings of the 2019 IEEE Energy Conversion Congress and Exposition (ECCE), Baltimore, MD, USA, 29 September-3 October 2019; pp. 4801-4807.

24. Van der Merwe, W. Natural Balancing of the 2-Cell Modular Multilevel Converter. IEEE Trans. Ind Appl. 2014, 50, 4028-4035. [CrossRef]

25. Lipo, T.; Holmes, D. Pulse Width Modulation for Power Converters: Principles and Practice; Wiley: Hoboken, NJ, USA, 2003.

Publisher's Note: MDPI stays neutral with regard to jurisdictional claims in published maps and institutional affiliations.

(C) 2020 by the authors. Licensee MDPI, Basel, Switzerland. This article is an open access article distributed under the terms and conditions of the Creative Commons Attribution (CC BY) license (http://creativecommons.org/licenses/by/4.0/). 